\title{
Squamous papillomatosis of the trachea and bronchi
}

\author{
B. T. LE ROUX, M. A. WILLIAMS, AND S. KALLICHURUM \\ From the University of Natal, Durban, South Africa
}

\begin{abstract}
A squamous papilloma which occluded the trachea of a young man was managed by resection at tracheotomy. Thereafter it was possible further to examine the bronchi bronchoscopically, and widespread papillomatosis confined to the left bronchi was seen. Following bronchography, which showed left-sided bronchiectasis, left pneumonectomy was undertaken. Case details are presented and the literature which relates to bronchial papillomatosis is reviewed.
\end{abstract}

The purpose of this paper is to present in detail the case report of a patient with tracheal and left bronchial papillomatosis and to outline what is known of this rare condition.

\section{CASE REPORT}

A Bantu man of 26 years presented with the history of ingravescent cough productive of purulent sputum for about a year. The features immediately apparent on clinical examination were wheeze, of tracheal origin, and mediastinal deviation to the left. Radiographically, the left lung was shrunken and partly airless (Fig. 1). At bronchoscopy a tumour in the trachea was seen. It lay at the level of the thoracic inlet and occluded almost completely the tracheal lumen. The appearances were reminiscent of villous papilloma of the bladder. An extensive biopsy was

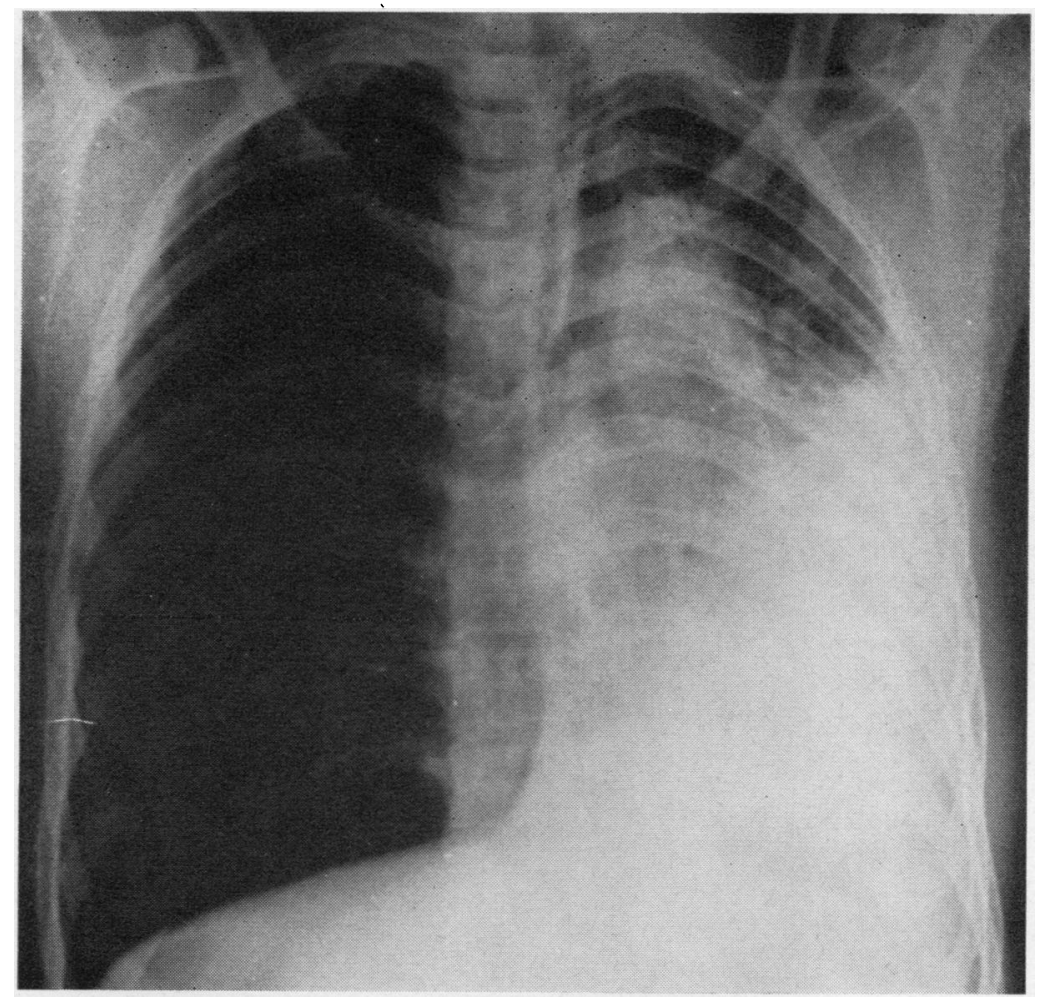

FIG. 1. Postero-anterior chest radiograph in which is shown shrinkage of the left lung with part of the left lower lobe airless and opaque. 


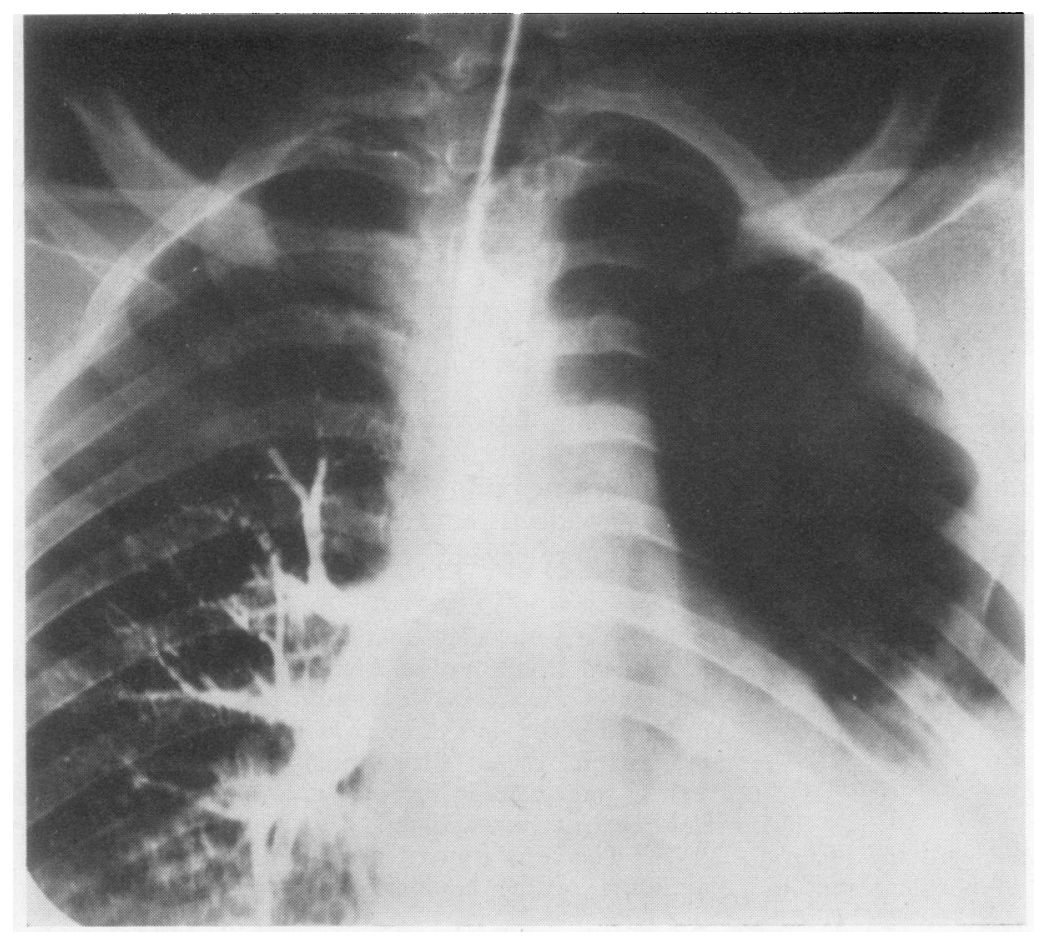

FIG. 2. The filling defect in trachea is visible close to the shadows of the sternal ends op the clavicle. Contrast medium has not entered the left stem bronchus.

FIG. 3. Bronchogram made 10 days after resection of the tracheal tumour, in which can be seen the filling defect produced by papillomata in the left upper and lower bronchi and left basal segmental bronchiectasis.

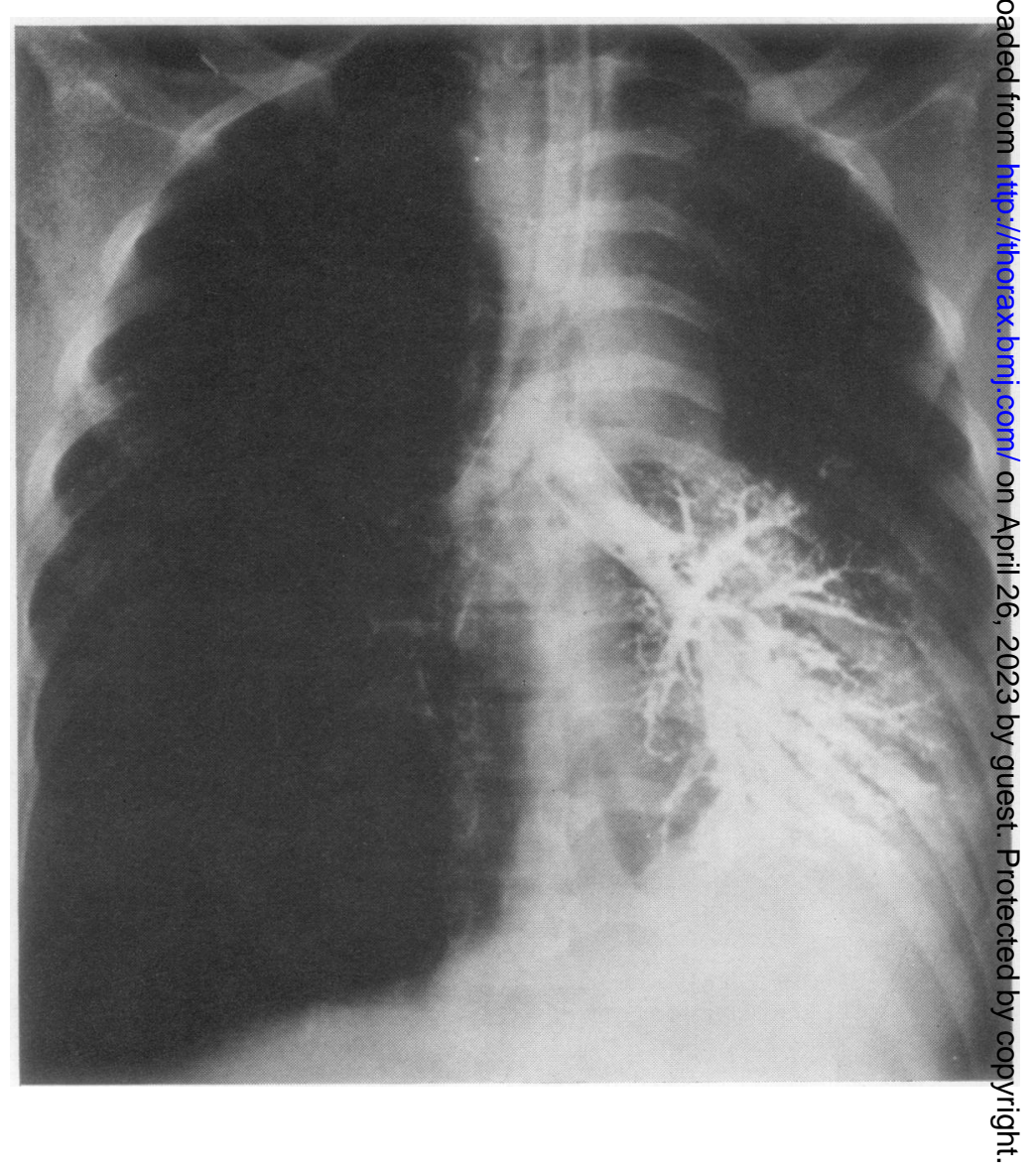


made, as much therapeutically as for diagnostic purposes, and the histological report on the resected material was that the tumour was a squamous papilloma. Thereafter a tracheogram was made (Fig. 2). Some right bronchi were outlined by contrast medium, and it was not known what interpretation to place on failure of the left bronchi to be outlined. It was judged most appropriate to resect the tracheal tumour at tracheotomy. As a preliminary to this a further attempt was made to introduce a bronchoscope past the tracheal tumour; but without success. At the time of tracheotomy, the state of the air passages beyond the tracheal tumour was not known.

Through a vertical mid-line incision, which included splitting the proximal half of the sternum, and which extended proximally to the thyroid cartilage, the trachea was exposed and almost completely transected at the level of the fifth cartilaginous ring. The fungating tumour sprouted from a relatively small pedicle and this was divided. The site of origin of the tumour was fulgurated and the trachea was repaired. At postoperative bronchoscopy multiple papillomata were seen to arise from the floor of the left stem bronchus, the most proximal, which was amputated with a biopsy forceps, close to the main carina and those arising more distally occluding almost completely the origins of the left upper and lower bronchi. The right bronchi were normal. There were considerable volumes of pus in the left stem bronchus.

Recovery from tracheostomy was uneventful. After an interval of 10 days a bronchogram was made (Fig. 3). The left upper bronchi were incompletely filled; the left basal bronchi were bronchiectatic. Some of the papillomata were evident as filling defects. It was elected at this juncture to proceed to left pneumonectomy, on these grounds: the multiplicity of the leftsided papillomata made endoscopic fulguration unlikely to be a successful manœuvre; shrinkage of the left lung was judged of long standing and this lung was thought likely to be the seat of chronic infection, the consequence of bronchial obstruction, left basal bronchiectasis having been demonstrated; the mode of spread of bronchial papilloma by implantation lent urgency to removal of all known papillomata, while the right bronchi were still known to be normal. Left pneumonectomy was therefore undertaken. The bronchi of the resected lung were opened and photographed (Fig. 4). The histology of the left bronchial papillomata was indistinguishable from that in the trachea; the characteristic features were a fibrous stroma covered by squamous epithelium (Fig. 5). Invasion of adjacent tissues was not demonstrated.

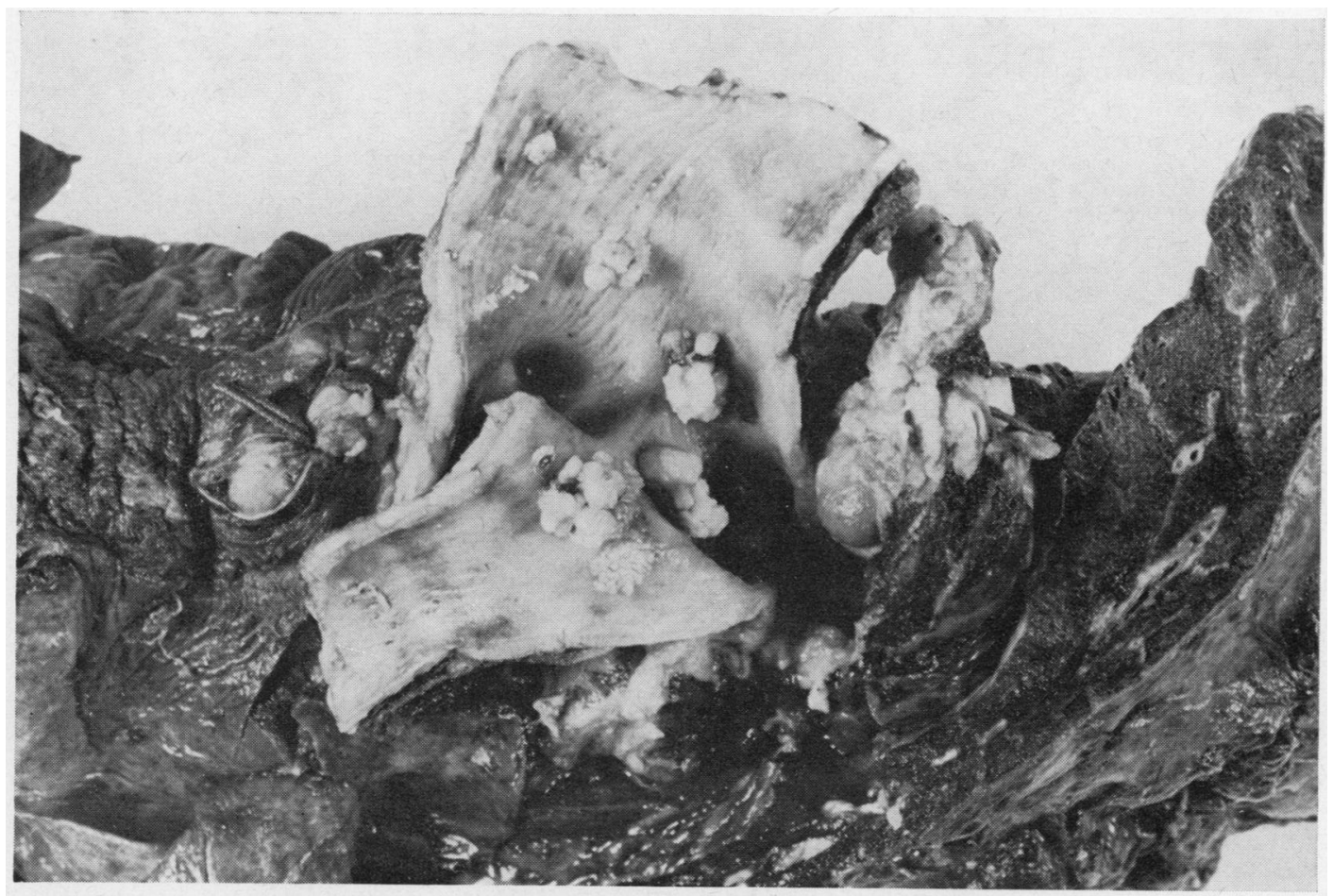

FIG. 4. Naked-eye specimen showing bronchial papillomata. 

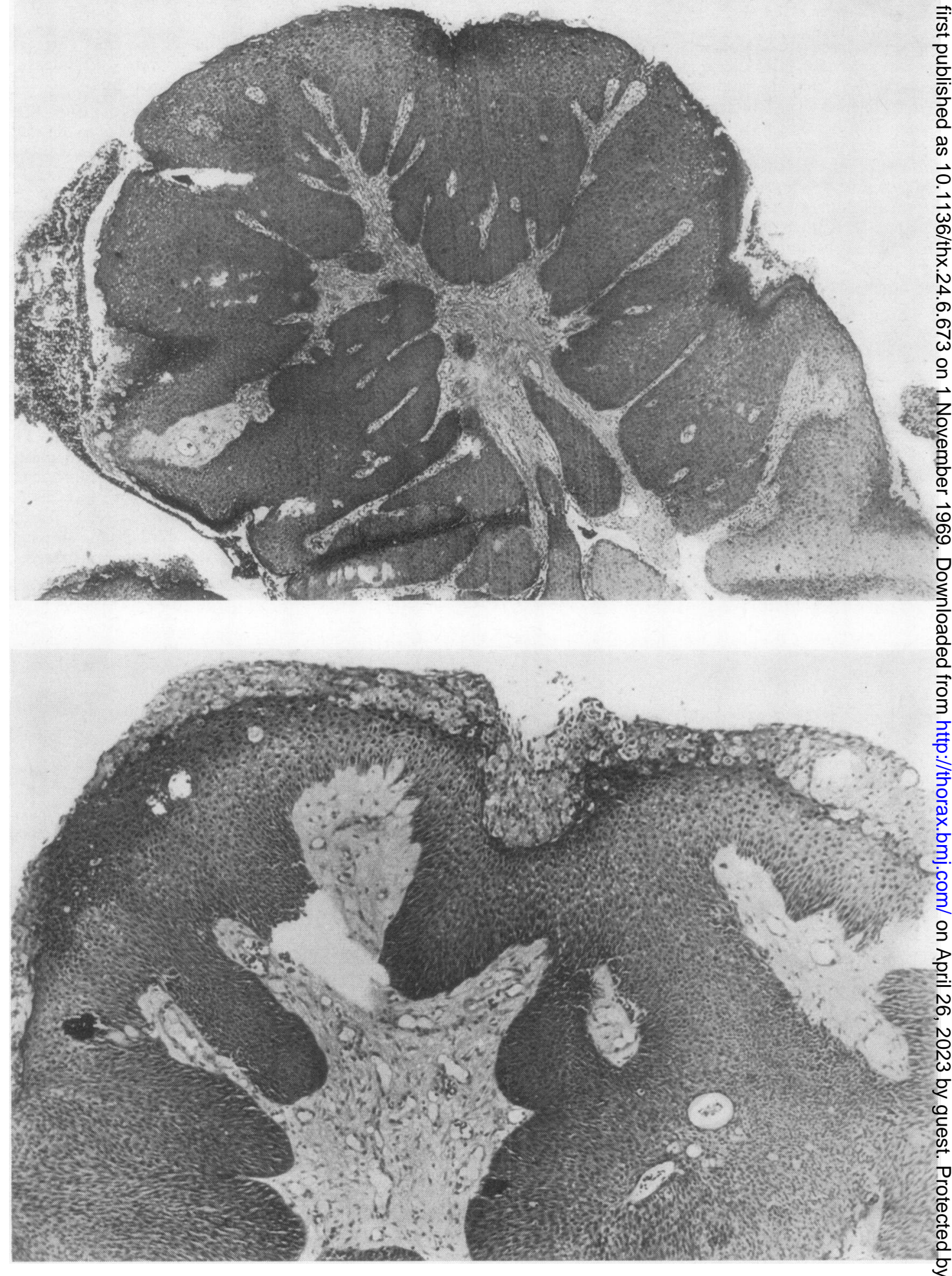

FIG. 5. (above) Low-power view of papilloma shows a fibrous stroma covered by hyperplastic squamous epithelium. (below) High-power view shows epithelial covering which consists of normal stratified squamous cells. 


\section{DISCUSSION}

Tracheal and bronchial papillomata are rare. Drennan and Douglas (1965) suggest that they should be classified as inflammatory, solitary, and multiple. Inflammatory papillomata show histologically the presence of an inflammatory reaction. They are usually, but not invariably, covered by respiratory epithelium. They may be single or multiple and are said to occur in association with inflammatory processes of long standing. Single benign papillomata are rare. Jackson and Hatch (1968) reported two cases and were able to find reference in the literature to only four previously recorded cases. Histologically, these show a fibrous tissue stroma covered with squamous epithelium, a pattern identical with that described when the tumours are multiple. Multiple papillomatacauliflower-like tumours arising in the larynx, trachea, and bronchi-are less rare. An extreme example reported by Kirchner (1951) was a case in which the larynx, trachea, and all the bronchi were completely filled with tumour. Ullmann (1923) was the first to suggest that squamous papillomata of the respiratory tract were the sequel to a virus infection, and this hypothesis is generally accepted. A viral aetiology explains, perhaps, the tendency of the tumours to recur and to implant throughout the respiratory tract. Implantation has resulted in the use of the term 'benign metastasizing papilloma'. To place in juxtaposition the adjectives benign and metastasizing is questionable, and outlines the difficulty often experienced in relating the histological and clinical behaviour of tumours. The histological criteria of malignancy have been demonstrated terminally in a case of multiple papillomatosis (Kaufman and Klopstock, 1963), and Smith and Dexter (1963) reported, in relation to papillary neoplasms of the bronchi, atypical cytological features which were interpreted as evidence of low-grade malignancy.

The case here reported is unusual in that the trachea and the bronchi of one side were involved without involvement of the opposite lung and the larynx. It remains to be seen if bronchial papillomata appear in the right bronchi. The patient is well one year after pneumonectomy.

\section{REFERENCES}

Drennan, J. M., and Douglas, A. C. (1965). Solitary papilloma of a bronchus. J. clin. Path., 18, 401.

Jackson, D. A., and Hatch, H. B. (1968). Solitary benign squamous papilloma of the bronchus. Amer. Rev. resp. Dis., 97, 699.

Kaufman, G., and Klopstock, R. (1963). Papillomatosis of the respiratory tract. Ibid., 88, 839.

Kirchner, J. A. (1951). Papilloma of the larynx with extensive lung involvement. Laryngoscope (St Louis), 61, 1022.

Smith, J. F., and Dexter, D. (1963). - Papillary neoplasms of the bronchus of low-grade malignancy. Thorax, 18, 340 .

Ullmann, E. V. (1923). On the aetiology of the laryngeal papilloma. Acta oto-laryng. (Stockh.), $5,317$. 